\title{
Cultural Adaptation and Psychometric Properties of the Persian Version of the Circumstances, Motivation, and Readiness Scale
}

\author{
Ensiyeh Norozi, ${ }^{1}$ Mohammad Reza Miri, ${ }^{2}$ Raheleh Soltani, ${ }^{3}$ Ahmad Ali Eslami, ${ }^{4,}$ Ali Reza Harivandi, ${ }^{5}$ \\ and Reza Dastjerdi ${ }^{6}$
}

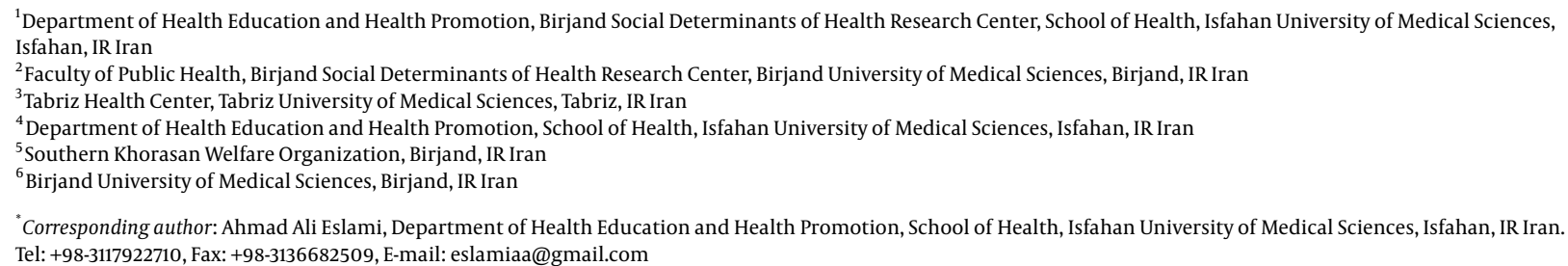
Tel: +98-3117922710, Fax: +98-3136682509, E-mail: eslamiaa@gmail.com

Received 2014 September 09; Revised 2015 February 25; Accepted 2015 April 26.

\begin{abstract}
Background: Treatment motivation has always been an important issue in substance abuse treatment. In recent decades, several instruments have been developed to measure this concept.

Objectives: In this study, cultural adaptation and psychometric properties of the Persian version of the circumstances, motivation and readiness scale (CMR) are illustrated in a sample of Iranian addicts.

Materials and Methods: The translation process followed Beaton et al.'s (2000) guideline for the cross-cultural adaptation of selfadministered questionnaires, including the steps of translation, synthesis, back translation, expert committee review, and pretesting. The final version of the Persian CMR was assessed for internal consistency and construct validity $(n=203)$.

Results: There was one eliminated item in the cross-cultural adaptation process. Also, four items that had low correlation with the total score were excluded from the questionnaire during the initial analysis. Using the remaining items, Principle axis factoring with Promax rotation was performed and three factors, circumstance, motivation, and readiness, were identified. The secondary order three factor model provided a good statistical and conceptual fit for the data. Internal consistency met the criterion for a reliable measure (Cronbach's alpha $=0.840$ ). The $\alpha$ range for these identified factors was 0.597 to 0.837 .

Conclusions: Although the CMR was originally designed for use in TC treatment, this study suggests that it is also applicable, with some modifications, in short-term residential camps. Also, it is concluded that the Persian translation of the CMR can be applied for studies among Persian addicts.
\end{abstract}

Keywords: Cultural Adaptation, Circumstances, Psychometrics, CMR

\section{Background}

Nowadays, the issue of substance related disorders has become a major social problem. Based on statistics released by the United Nations office on drugs and crime (UNODC), about 230 million people, or 5 percent of the world's adult population (persons age 15 - 64 years old), are estimated to have used an illegal drug at least once in 2010. According to this report, about one percent of deaths among adults are attributed to illegal drug use (1). In Iran, after disasters, addiction has the highest burden of diseases among men, and the number of addicts is about 1.2 million (2).

Important advances in the treatment of addiction have been made in recent decades (3), but, despite these advances, a major problem in the treatment of addicts, even with prolonged abstinence period, is their high rate of relapse (4). For example, the six-month relapse rates following various classes of substance use cessation attempts generally range from 45\% - 65\% (5-7).

Numerous studies have investigated factors influencing relapse among different groups of substance users. Based on the results of some studies, low motivation to quit is one of the most important predictors of relapse among addicts $(6,8-10)$. Indeed, treatment motivation is considered to be one of the most important predictors of treatment engagement and the ability to benefit from treatment $(11,12)$.

In line with the growing acceptance of motivation as an essential factor for effective treatment, several instruments have been developed to measure motivation in the field of substance abuse, including: the 
stages of change readiness and treatment eagerness scale (SOCRATES), which was developed with alcohol abusers (13); the Circumstances, motivation, readiness, and suitability scales (CMRS), which was developed with drug abusers in therapeutic community treatment centers and subsequently used with both adult and adolescent drug abusers (14); and the Texas Christian University motivational assessment instrument, which has been used with a variety of drug-abusing patients and measures problem recognition, desire for help, and treatment readiness (15). Only limited efforts have been undertaken in Iran to translate these instruments and to determine their reliability and validity among Iranian addicts.

The CMR scale is an 18-item, self-administered questionnaire designed to assess motivation among abusers of illegal substances. This instrument is based on the CMRS, in which the suitability (S) scale was eliminated and the other scales were shortened (16). The instrument features Likert-type responses rated on a 5-point scale reflecting the extent of agreement with the respective items ( $1=$ strongly disagree to $5=$ strongly agree). The items in the $\mathrm{C}$ scale refer to external conditions that influence decisions to enter or remain in treatment (circumstances 1) or to leave treatment (circumstances 2). The M scale refers to a client's inner reasons for change, while the R scale measures the individual's perceived need for treatment, as opposed to selftreatment or other self-change choices (17). The CMR scores have been found to be directly and positively related to treatment retention, and have been found to be indirectly related to treatment outcomes (17). Meanwhile, this instrument has good predictive validity (18).

\section{Objectives}

Considering the importance of motivation in treatment outcomes, the utility of reliable and valid measures is essential for applying this concept in studies among Iranian addicts. For this reason, this study is focused on translating the CMR scale into the Persian language and to adapting it to Iranian culture, so that we may assess its reliability and validity among Iranian addicts.

\section{Materials and Methods}

This study was performed in two stages. In the first stage, the questionnaire was translated into the Persian language and culturally adapted to Iranian culture. In the second stage, it was tested among addicted referred to addiction treatment short-term residential camps (length of residence: 21 - 30 days) to assess internal consistency and construct the validity of the scale.

\subsection{Stage 1: Cross-Cultural Adaptation}

The cross-cultural adaptation was performed based on Beaton et al.'s (2000) guide for the cross-cultural adaptation of self-administered questionnaires. It recommends a five-step process for cross-cultural adaptation: translation, synthesis, back translation, expert committee review, and pre-testing (19).

\subsubsection{Step 1: Initial Translation}

The initial translation into Persian was performed by two bilingual Iranian translators, one of whom was a psychologist and other a health education and promotion specialist. As Beaton's guide noted, one of the translators should be aware of the concepts being examined in the questionnaire being translated, and the other should neither be aware nor informed of the concepts being quantified (19). In this study, the psychologist was familiar with the scale's concepts.

\subsubsection{Step 2: Synthesis of the Translations}

In the next step, the two translators and the researchers synthesized the results of the two translations into one common translation based on the original scale and resolved any discrepancies with the translators' reports.

\subsubsection{Step 3: Back Translation}

Next, this common adapted version was backtranslated into English by two bilingual native-speaking English translators who worked independently. They also were neither aware of nor informed of the concepts being explored.

\subsubsection{Step 4: Expert Committee}

In step 4, an expert committee consisting of five experts, two health educators, one psychometrist, and two translators (forward and back translators), reviewed and compared the common translation, the two back translations, and the original scale to reach consensus on discrepancies and produce a pre-final Iranian version of the CMR.

Then, a panel of experts consisting of ten experts, four health educators, four clinical psychologists, and two addiction treatment physicians, was convened. The content validity of the questionnaire, via the content validity ratio (CVR) and content validity index (CVI), was determined.

\subsubsection{Calculating the Content Validity Ratio}

To calculate the CVR, the experts rated each item of the questionnaire on a Likert-type ordinal scale ( $1=$ it is essential, 2 = it is useful but not essential, $3=$ it is not essential). The calculated ratios for each item were compared with the 
numbers provided by Lawsche (20). If the calculated value was greater than the number given in Lawsche's table (for this study, CVR values higher than 0.62), the item was considered as an appropriate and necessary one and was retained for subsequent analysis.

\subsubsection{Calculating the Content Validity Index}

CVI amounts can be calculated for each item (Item-CVI) based on Lynn's pattern (21), or they can be determined as a total amount for each instrument (scale-CVI), based on Polit et al. work (22). In this study, Lynn's descriptive method was used, and the experts rated simplicity $(1=$ not simple to $4=$ very simple), relevance $(1=$ not relevant to $4=$ very relevant), and clarity ( $1=$ not clear to $4=$ very clear $)$ for each item on a Likert-type ordinal scale. Based on Lynn's guidelines, if the number from the expert panel was 5 or less than 5 , the content validity index for each item should be 1 , and if the number from the experts was 6 or more than 6 , the index should not be less than 0.78 (21).

\subsubsection{Step 5: Test of the Pre-final Version}

The final stage of adaptation process was the pretest. Beaton's recommendation of a minimum sample of 30 subjects was followed (19), and pre-testing was conducted among 30 addicted men who had been referred to an addiction treatment short-term residential camp. Pretesting was performed in order to ensure whether the target group could understand the adapted version properly.

Following the pretest, a final version of questionnaire was prepared for field testing in a cross-sectional study. The purpose of this cross-sectional study was to evaluate the psychometric properties of the questionnaire.

\subsection{Stage 2: Determining the Psychometric Properties of the In- strument}

In this stage, the final questionnaire that was developed in the previous stage was examined in terms of its features, such as construct validity and internal consistency in a cross-sectional study.

\subsubsection{Participants}

The study population was comprised of addicts referred to the Southern Khorasan province's addiction treatment short-term residential camp. Southern Khorasan is one of the 31 provinces of Iran and it borders Afghanistan (a country with the greatest rate of poppy cultivation and natural drugs in the world). Inclusion criteria were the main and primary diagnosis of substance dependency disorders, according to diagnostic criteria from the DSM-IV-TR and completion of a detoxification stage. Regular use of anti-psychotic drugs and an inability to answer questions due to physical and/or psychological problems were exclusion criteria. Meanwhile, all participants were informed about the study procedures and signed an informed consent form.

\subsubsection{Procedures}

The study sample size was estimated, as proposed by Bentler and Chou, a minimum of five subjects and maximum of ten subjects for each parameter (23). To test the psychometric properties of the Persian final version of the CMR, a convenience sample of 203 addicts was selected from all addicts referred to the treatment camps from May 2013 through November 2013. The CMR is a selfadministered scale, and each addict completed it individually with no assistance from the researchers. Instructions for completing the questionnaire were available in its front page.

\subsubsection{Data Analysis}

All analyses were performed using SPSS 15.0 and AMOS 18.0. Principal axis factoring was used to estimate the number of principal components and the factorability of the correlation matrices. Before conducting any exploratory factor analyses, internal consistency of the instrument was assessed using Cronbach's alpha. Our criterion for verifying the reliability of the instrument was Cronbach's $\alpha$ greater than 0.70. Meanwhile, the corrected item total correlation was also examined, and items whose correlation with the total score was less than 0.3 were excluded from questionnaire. To ensure the adequacy of the sample size, the Kaiser-Meyer-Olkin (KMO) measure of sampling adequacy was performed. If the KMO value is equal to or greater than 0.6, the sample size is adequate. Bartlett's test of sphericity was also examined. Then, principal axis factoring using non-orthogonal promax rotation procedure $(K=4)$ was conducted. The factor structure was assessed using several criteria, including (a) analysis of the Eigen values greater than 1 in the Scree plot, (b) item cut-off loading greater than or equal to 0.40 , and (c) parallel analysis by Mont Carlo PCA (24).

In addition, confirmatory factor analysis was employed to assess the factorial validity of the CMR. The following indices were used to evaluate model fit, as recommend by Hu et al (1999) (25): comparative fit index (CFI), the Tucker-Lewis index (TLI), root mean squared error of approximation (RMSEA), the normed $\chi^{2}\left(\chi^{2} / \mathrm{df}\right)$, and Parsimonious Normed Fit Index (PNFI). The following cut-offs were used for acceptable fit: CFI $\geq 0.90$, TLI $\geq 0.90$, RMSEA $\leq 0.08$, normed $\chi^{2}<5$, and PNFI $\geq 0.50(25)$. 


\section{Results}

\subsection{Descriptive Analysis}

Descriptive statistics were used to summarize study the participants' demographic information and other psychosocial variables. The mean age of participants was $29.09 \pm 6.86$ (15-55 years) years. The majority of them (97\%) were male, and the average number of relapses was $2.84 \pm$ 3.93. Sample characteristics are shown in Table 1.

Table 1. Demographic Characteristics of the Participants $(n=203)$

\begin{tabular}{|c|c|}
\hline Characteristics & No. (\%) \\
\hline \multicolumn{2}{|l|}{ Age, $y$} \\
\hline$<20$ & $18(8.9)$ \\
\hline $20-25$ & $57(28.1)$ \\
\hline $25-30$ & $53(26.1)$ \\
\hline $30-35$ & $36(17.7)$ \\
\hline $35>$ & $39(19.2)$ \\
\hline \multicolumn{2}{|l|}{ Marital status } \\
\hline Single & $108(53.2)$ \\
\hline Married & $87(42.9)$ \\
\hline Divorced or separated & $8(3.9)$ \\
\hline \multicolumn{2}{|l|}{ Educational level } \\
\hline$<$ High school & $113(55.6)$ \\
\hline High school or more & $90(44.4)$ \\
\hline \multicolumn{2}{|l|}{ Occupation status } \\
\hline Unemployed & $103(50.7)$ \\
\hline Employed part-time & $72(35.5)$ \\
\hline Employed full- time & $28(13.8)$ \\
\hline \multicolumn{2}{|l|}{ Substance type } \\
\hline Opiate & $57(28.1)$ \\
\hline Stimulants & $80(39.4)$ \\
\hline Hallucinogens & $9(4.4)$ \\
\hline Multiple drugs & $57(28.1)$ \\
\hline
\end{tabular}

\subsection{Cultural Adaptation}

In the translation, synthesis and back translation steps there were no discrepancies between translations. So all items were retained for expert review and calculating content validity. By the expert review committee, question 3 "I believe that my family/relationship will try to make me leave treatment after a few months" changed to "I believe that my family/relationship will try to make me leave treatment after a few days". To determine CVR, necessity of each item was reviewed by expert panel and since CVR values for all items was higher than $\mathbf{0 . 6 2}$, so all items were retained for subsequent analysis. Then for determining CVI, relevant, clarity and simplicity criteria of each item was reviewed and higher values of 0.79 was accepted. According to the index, all 18 items were retained for the next stage.

In the pre-testing, respondents had difficulties to answer question 18: "I'm willing to enter treatment as soon as possible". So this item was removed.

\subsection{Cross-Sectional Study}

\subsubsection{Exploratory Factor Analysis}

Before conducting an exploratory factor analysis, the internal consistency of the questionnaire was examined using the Cronbach's Alpha test $(\alpha=0.797)$. Meanwhile, the Corrected Item Total Correlation values for four items $(4,5$, 6 , and 12) were less than 0.3 , so these items, all of which had a low correlation with the total score, were excluded from the questionnaire.

Using the remaining items, principle axis factoring with Promax rotation was performed. The correlation matrix was considered to be factorable (KMO $=0.864$, Bartlett's test of sphericity $=784.882, \mathrm{P}<0.000)$. An exploratory factor analysis produced three factors with Eigen values greater than 1 . The Scree plot also indicated the three factors solution (Figure 1). In total, these three factors explained $55.39 \%$ of the extracted variances. This result was not obtained by the parallel analysis that was performed by Mont Carlo software. Indeed, PA retained two factors, accounting for $47.24 \%$ of the total item variances, but since the three factors structure was more consistent with the theoretical foundations of the research, this structure was maintained. Item loadings from PCA of the culturally adapted CMR are shown in Table 2 . The pattern matrix indicated that factor 1 contained all the circumstances (1) items from the original scale; factor 2 contained all the motivation items, in addition to items 14 and 15 from the original readiness scale. Factor 3 consisted of all readiness items from the original scale, excluding items $12,14,15$, and 18. As is noted, all of the circumstances (2) items from the original scale were excluded from the Persian version of the CMR.

In general, the circumstances factor is comprised of three items, the motivation (M) factor is comprised of seven items, and the readiness ( $R$ ) factor is comprised of three items. All factors were unidimensional.

\subsubsection{Confirmatory Factor Analysis}

Based on the results of the exploratory factor analysis, the hypothesized measurement model included circumstances as latent variable with three items as indicators, motivation as a latent variable with seven items as indicators, and readiness as a latent variable with three items as 


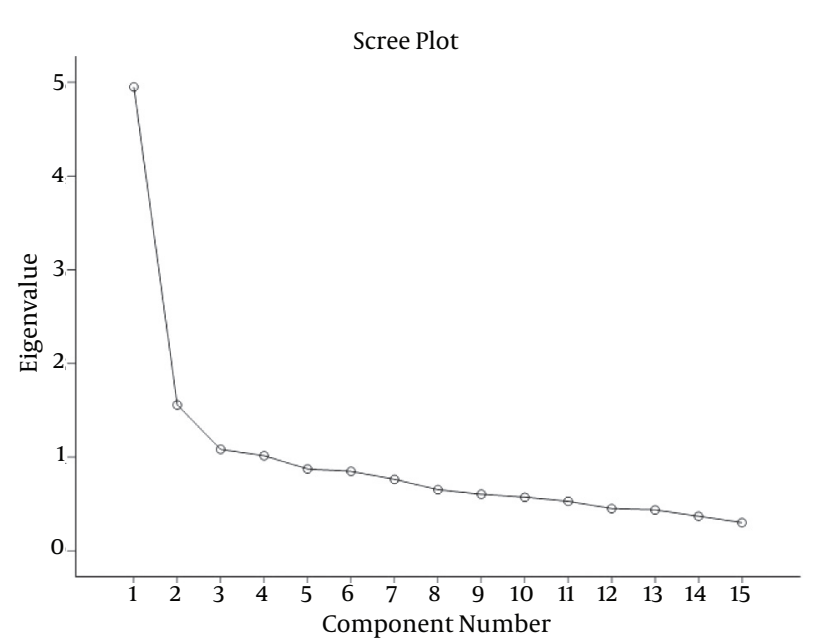

Figure 1. Scree Plot for Principal Axis Factoring of the Culturally Adapted Circumstances, Motivation, and Readiness Scale (CMR; $\mathrm{n}=203$ )

Table 2. Item Loadings From Principal Axis Factoring of the Culturally Adapted Circumstances, Motivation, and Readiness Scale $(\mathrm{CMR} ; \mathrm{n}=203)^{\mathrm{a}}$

\begin{tabular}{|c|c|c|c|}
\hline Item & Motivation (M) & Readiness (R) & Circumstances (C) \\
\hline 8 & 0.832 & - & - \\
\hline 11 & 0.770 & - & - \\
\hline 10 & 0.764 & - & - \\
\hline 7 & 0.713 & - & - \\
\hline 14 & 0.614 & - & - \\
\hline 9 & 0.592 & - & - \\
\hline 15 & 0.520 & - & - \\
\hline 16 & - & 0.898 & - \\
\hline 13 & - & 0.696 & - \\
\hline 17 & - & 0.662 & - \\
\hline 3 & - & - & 0.909 \\
\hline 1 & - & - & 0.849 \\
\hline 2 & - & - & 0.492 \\
\hline \% Variance & 36.85 & 10.39 & 8.14 \\
\hline Cronbach's $\alpha$ & 0.837 & 0.660 & 0.597 \\
\hline Mean \pm SD & $30.18 \pm 4.43$ & $11.91 \pm 2.38$ & $11.40 \pm 2.82$ \\
\hline
\end{tabular}

indicators. The three latent variables were hypothesized to serve as indicators of a higher-order CMR latent factor (Figure 2). CFA results indicated that the data had good fit with the secondary order three factor model, and fit indices were acceptable (Table 3, report indices).

\subsubsection{Reliability}

Regarding reliability, Cronbach's alpha for the overall scale was good ( $\alpha=0.840)$. A similarly high alpha coefficient was obtained for the M scale $(\alpha=0.837)$, but lower alphas were obtained for the R scale $(\alpha=0.660)$ and the $\mathrm{C}$ scale $(\alpha=0.597)$.

\subsubsection{Correlation Analysis}

Interclass Pearson correlation was used to calculate correlations between subscales and Total CMR. As shown in Table 4, factors were found to be significantly correlated with each other and with the total score $(\mathrm{P}<0.01)$.

\section{Discussion}

This study examined the cultural adaptation and psychometric properties of a Persian translation of the CMR.

There was one eliminated item in the pre-testing process. This item "I'm willing to enter treatment as soon as possible" was originally related to the readiness scale. This item was not applicable for the target group of this study, because at the time of completing the questionnaire, treatment had already begun for this group.

As noted, four items $(4,5,6$, and 12$)$ were excluded from the questionnaire in the early stage of analysis, due to low correlation with the total score. These items, excluding item 12 , originally were related to circumstances, which measures individuals' concerns about financial, family, and other problems that could potentially be an obstacle to continued treatment (item 4: "I believe that my family/relationship will try to make me leave treatment after a few days," item 5: "I am worried that I will have serious money problems if I stay in treatment," and item 6: "I feel I have too many outside problems that will prevent me from completing treatment"). The omission of these items may be explained by the heterogeneity inherent in these items for the target group of this study. Indeed, these items were originally developed for use in TC treatment and have little in common with short-term residential camps, because addicts referred to the camps, due to their short stays, do not typically have concerns about financial, family, and other problems.

In this study, exploratory factor analysis revealed three factors, all of which were unidimensional. These findings are not in line with the results of other studies $(16,18)$. Indeed, the findings from American and Dutch studies identified one bidimensional (circumstances) and two unidimensional (motivation and readiness) scales $(16,18)$. This finding may probably be explained by omission of circumstances scale in the early stage of analysis. On account of these omissions, the bidimensional structure of the $\mathrm{C}$ scale has been converted to a unidimensional structure. 


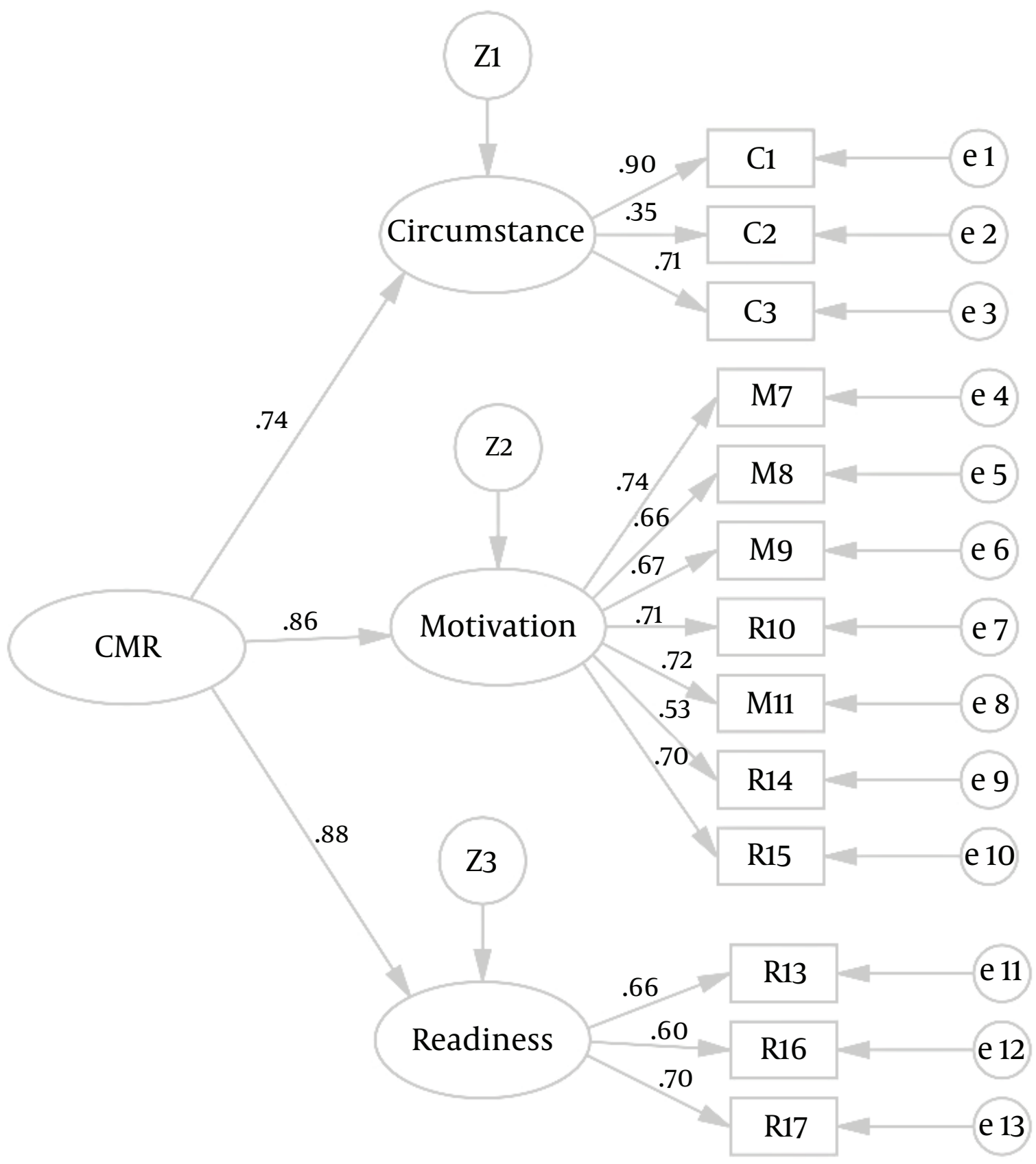

Figure 2. Confirmatory Factor Analysis (CFA) of the Culturally Adapted Circumstances, Motivation, and Readiness scale (CMR; $n=203$ )

The confirmatory factor analysis results confirmed that the data had good fit with the three component scale. This model's fit was better than that reported in the Soyez et al. study (18). In fact, the results of the psychometric evaluation of the Dutch translation of the CMR indicated that model's fit was modest (18).
The alpha coefficient for the motivation scale was the highest (0.83). Other coefficients ranged from 0.59 (circumstance) to 0.82 (overall scale). The internal consistency of the $C$ scale was limited, because this scale consisted of items measuring different external conditions, such as legal and family conditions, that did not necessarily change 
Table 3. Fit Indices for Confirmatory Factor Analysis of the Culturally Adapted Circumstances, Motivation, and Readiness scale (CMR; $n=203$ )

\begin{tabular}{lcccccc}
\hline & NPAR & df & RMSEA & TLI & CFI & PNFI \\
\hline CMR (3 factors) & 41 & 63 & 0.063 & 0.92 & 0.93 & 0.7 \\
\hline $\begin{array}{l}\text { Abbreviations: CFI, Comparative Fit Index; df, degrees of freedom; } \\
\text { Index (TLI); } \chi \chi^{2} / \mathrm{df} \text {, normed } \chi^{2} .\end{array}$
\end{tabular}

Table 4. Correlations Between the 13-Item Culturally Adapted Circumstances, Motivation, and Readiness Scale (CMR) and Total Scores ${ }^{\mathrm{a}}$

\begin{tabular}{lccc}
\hline & Circumstance & Motivation & Readiness \\
\hline Circumstance & 0.406 & 0.708 & 0.367 \\
Motivation & & 0.556 \\
Readiness & & 0.890 \\
\hline
\end{tabular}

${ }^{\mathrm{a}}$ For all values $\mathrm{P}<0.01$.

together. This finding is in line with results of other studies. These studies also identified the lowest coefficient for the C-scale (0.41 - 0.44 in an American population, and 0.54 in a Flemish sample) $(16,18)$.

Some limitations of this study must be acknowledged. The process of cultural adaptation and psychometric analysis of the CMR resulted in some omissions in the scale. These omissions may limit comparisons between the results of this version of the CMR with those of other studies that used the complete scale. Another weakness of the study is that the current analyses do not provide comprehensive coverage of both sexes, and the results are largely limited to males. Thus, a cross-validation of the CMR in females is necessary before making any claims regarding the generalizability of the instrument to both sexes.

In conclusion, the CMR appears to be a valid instrument for use in Iranian samples. Although the CMR was originally designed for use in TC treatment, this study suggests that it also is applicable, with some modifications, in short-term residential camps. Additional research should be conducted among different groups of addicts receiving different treatment modalities to define the broader applicability of the instrument.

\section{Acknowledgments}

The deputy of research and technology of Isfahan University of Medical Sciences supported this study. The authors wish to thank the Southern Khorasan province's welfare organization for its cooperation with the project, and especially the patients for their participation.

\section{References}

1. United Nations Office on Drugs and Crime. . World drug report 2012. New York: United Nations Office on Drugs and Crime; 2012.
2. Narenjiha H, Rafiey H, Baghestani A. Rapid situation assessment of drug abuse and drug dependence in Iran. Tehran: DARIUS Institute; 2007.

3. Kimberly JR, McLellan AT. The business of addiction treatment: A research agenda. J Subst Abuse Treat. 2006;31(3):213-9. doi: 10.1016/j.jsat.2006.06.018. [PubMed:16996384].

4. Yan Y, Nabeshima T. Mouse model of relapse to the abuse of drugs: procedural considerations and characterizations. Behav Brain Res. 2009;196(1):1-10. doi: 10.1016/j.bbr.2008.08.017. [PubMed: 18782591].

5. McLellan AT, McKay JR, Forman R, Cacciola J, Kemp J. Reconsidering the evaluation of addiction treatment: from retrospective follow-up to concurrent recovery monitoring. Addiction. 2005;100(4):447-58. doi: 10.1111/j.1360-0443.2005.01012.x. [PubMed: 15784059].

6. Mohammadpoorasl A, Fakhari A, Akbari H. Addiction Relapse and Its Predictors: A Prospective Study. J Addict Res Ther. 2012;03(01) doi: 10.4172/2155-6105.1000122.

7. Siegal HA, Li L, Rapp RC. Abstinence trajectories among treated crack cocaine users. Addict Behav. 2002;27(3):437-49. [PubMed: 12118630].

8. Cornelius JR, Maisto SA, Pollock NK, Martin CS, Salloum IM, Lynch KG, et al. Rapid relapse generally follows treatment for substance use disorders among adolescents. Addict Behav. 2003;28(2):381-6. [PubMed: 12573689].

9. Gossop M, Stewart D, Browne N, Marsden J. Factors associated with abstinence, lapse or relapse to heroin use after residential treatment: protective effect of coping responses. Addiction. 2002;97(10):1259-67. [PubMed: 12359030].

10. Laudet AB, Stanick V. Predictors of motivation for abstinence at the end of outpatient substance abuse treatment. J Subst Abuse Treat. 2010;38(4):317-27. doi:10.1016/j.jsat.2010.01.007. [PubMed: 20185267].

11. Austin A, Hospital M, Wagner EF, Morris SL. Motivation for reducing substance use among minority adolescents: targets for intervention. J Subst Abuse Treat. 2010;39(4):399-407. doi:10.1016/j.jsat.2010.07.008. [PubMed: 20822879].

12. Goodman I, Peterson-Badali M, Henderson J. Understanding motivation for substance use treatment: the role of social pressure during the transition to adulthood. Addict Behav. 2011;36(6):660-8. doi: 10.1016/j.addbeh.2011.01.011. [PubMed: 21295918].

13. Miller WR, Tonigan JS. Assessing drinkers' motivation for change: The Stages of Change Readiness and Treatment Eagerness Scale (SOCRATES). Psychol Addict Behav. 1996;10(2):81-9. doi: 10.1037/0893164X.10.2.81 
14. De Leon G, Jainchill N. Circumstance, motivation, readiness and suitability as correlates of treatment tenure. J Psychoactive Drugs. 1986;18(3):203-8. doi: 10.1080/02791072.1986.10472348. [PubMed: 3772644].

15. Joe GW, Simpson DD, Broome KM. Effects of readiness for drug abuse treatment on client retention and assessment of process. Addiction. 1998;93(8):1177-90. [PubMed: 9813899].

16. De Leon G, Melnick G, Kressel D, Jainchill N. Circumstances, motivation, readiness, and suitability (the CMRS scales): predicting retention in therapeutic community treatment. Am J Drug Alcohol Abuse. 1994;20(4):495-515. [PubMed: 7832182].

17. Leon GD, Melnick G, Hawke J. The motivation-readiness factor in drug treatment implications for research and policy. Advances Med Sociol. 2000;7:103-29. doi: 10.1016/s1057-6290(00)80006-6.

18. Soyez V, De Leon G, Rosseel Y, Broekaert E. Motivation and readiness for therapeutic community treatment: psychometric evaluation of the Dutch translation of the Circumstances, Motivation, Readiness, and Suitability scales. J Subst Abuse Treat. 2006;30(4):297-308. doi: 10.1016/j.jsat.2006.02.007. [PubMed:16716844].
19. Beaton DE, Bombardier C, Guillemin F, Ferraz MB. Guidelines for the process of cross-cultural adaptation of self-report measures. Spine (Phila Pa 1976). 2000;25(24):3186-91. [PubMed: 11124735].

20. Lawshe CH. A Quantitative Approach to Content Validity. Personnel Psychol. 1975;28(4):563-75. doi: 10.1111/j.1744-6570.1975.tb01393.x.

21. Lynn MR. Determination and quantification of content validity. Nurs Res. 1986;35(6):382-5. [PubMed: 3640358].

22. Polit DF, Beck CT, Owen SV. Is the CVI an acceptable indicator of content validity? Appraisal and recommendations. Res Nurs Health. 2007;30(4):459-67. doi:10.1002/nur.20199. [PubMed: 17654487].

23. Bentler PM, Chou CP. Practical Issues in Structural Modeling. Sociol Methods Res. 1987;16(1):78-117. doi:10.1177/0049124187016001004.

24. Watkins MW. Determining parallel analysis criteria. J Modern Appl Statistic Methos. 2005;5(2):8.

25. Hu LT, Bentler PM. Cutoff criteria for fit indexes in covariance structure analysis: Conventional criteria versus new alternatives. Structur Equation Modeling Multidisciplinar J. 1999;6(1):1-55. doi: 10.1080/10705519909540118. 